\title{
Festive flu delay ... and other stories
}

\begin{abstract}
A recent Minerva article (BMJ 2017;356:j54, doi:10.1136/bmj. j54) on antihypertensives and acute kidney injury inaccurately stated the results. The statement should have said "Compared with patients without chronic kidney disease, having stage 4 chronic kidney disease increased the risk of acute kidney injury by a factor of 6-8 times (independent of antihypertensive use)." Although the risk of acute kidney disease was slightly increased
\end{abstract}

for the entire study population during time exposed to renin-angiotensin system inhibitors (overall incidence rate ratio $1.12,95 \%$ confidence interval 1.07 to 1.17 ), among those with stage 4 chronic kidney disease it was actually reduced, compared with time exposed to other antihypertensives (incidence rate ratio $0.66,95 \% \mathrm{CI} 0.44$ to 0.97 ). 